\title{
Über die Lebensspanne hinweg: Bildung und Erziehung in professioneller Ausgestaltung durch Psychodrama
}

\author{
Stefan Gunkel • Birgit Szczyrba
}

In der Zielperspektive einer modernen, westlichen geprägten Erziehung plant und gestaltet der mündige, eigenständig handelnde und emanzipierte Mensch sein Leben. Individuelle Kompetenzentwicklung, differenzierte Handlungsfähigkeit, mündige Selbstbestimmtheit und Emanzipation erfordern zu ihrer Entwicklung das Eingebundensein in soziale Beziehungen. Bildung wird vom direkten sozialen Umfeld weg in einen größeren gesellschaftlichen Kontext gerückt. Aber Bildung ist häufig auch oder zunächst Erziehung. Ohne das eine ist das andere weniger wirksam oder gar nicht möglich. In vielen Sprachen gibt es deshalb nur ein Wort für beide Begriffe, z. B. das englische ,education“.

Wie kann das Psychodrama, sein Wert und seine Wirkungskraft, für professionelles Handeln im Erziehungs- und Bildungsbereich fruchtbar gemacht werden? J.L. Moreno hat ein reichhaltiges theoretisches Werk und ein Ensemble an Instrumenten hinterlassen, das heute im Sinne von ,paideia' Lern- und Entwicklungsprozesse des Menschen über die gesamte Lebensspanne hinweg begleiten kann. Erziehung und Bildung, auch Unterricht und Hilfe bei biografischen Brüchen oder schwierigen Lebenslagen, sind heutige Aufgabenfelder pädagogischer und sozialer Berufe. Sie haben die Bewältigung des Generationenunterschiedes zur Aufgabe, befördern die Gestaltung des Menschen zum Menschen, vermitteln Qualifikationen für eine arbeitsteilige Gesellschaft und kümmern sich um Menschen in Not.

Die in diesem Themenheft zusammen getragenen Beiträge bilden die professionelle Ausgestaltung von Erziehung und Bildung in den Praxisfeldern Schule, Hochschule, Kirche und freier Weiterbildung ab und zeigen auf, wie das Psychodrama in Hintergrund und Praxis zur Gestaltung von Lern- und Entwicklungsprozessen beiträgt.

\footnotetext{
Online publiziert: 18.01 .2011

(C) VS Verlag für Sozialwissenschaften 2011

Dipl.-Psych. S. Gunkel $(\bowtie)$

Psychiatrie Langenhagen, Klinikum Region Hannover,

Rohdehof 3, 30853 Langenhagen, Deutschland

E-Mail: stefan.gunkel@krh.eu

Dr. paed. B. Szczyrba

Qualitätsoffensive Exzellente Lehre, Projekt ,Educational Diversity“,

Fachhochschule Köln, Claudiusstr. 1, 50678 Köln, Deutschland

E-Mail: birgit.szczyrba@fh-koeln.de
} 
Christoph Hutter geht im einleitenden philosophisch ausgerichteten Artikel der Frage nach, welche wegweisenden Äußerungen zum Thema Erziehung im Werk von Moreno enthalten sind. Dieser sieht z. B. den Menschen grundsätzlich nicht als Objekt pädagogischer oder therapeutischer Interventionen, sondern als kreatives Wesen mit Bedürfnissen nach Beziehung und Potentialentfaltung, das ausgestattet ist mit Lebenshunger und Erkenntnisinteresse. Menschen existieren in bzw. durch soziale Netzwerke (Bedeutung der Gruppe als sozioemotionales Beziehungsgeflecht) und sie handeln in Rollen. Erziehung ist im Wesentlichen Beziehungsangebot, also v. a.,Begegnung' als ,höchster Form der Kommunikation“. Hutter vergleicht Grundannahmen, die Morenos Ansatz kennzeichnen, mit Konzepten von Martin Buber (,Ich und Du'), John Dewey (,learning by doing') Hartmut von Hentig (,Lernen in Gemeinschaft') und neurobiologischen Sichtweisen (Manfred Spitzer, Gerald Hüther).

Auch der Beitrag von Ferdinand Buer reflektiert das Werk Morenos, das im Kern Beziehungsarbeit ist, auf philosophischer Ebene, mit besonderer Bezugnahme auf die amerikanische Schule des Pragmatismus nach Dewey. Das Ergebnis eines Dialogs zwischen der impliziten Bildungstheorie Morenos und der expliziten Deweys wird an fünf Dimensionen eines umfassenden Bildungsprozesses in der Schule verdeutlicht: Erfahrungslernen, Ästhetik, Spiritualität, Ethik und Politik.

Das Psychodrama bietet wertvolles Potential, den Wandel des Lehrberufs an Schulen zu unterstützen. Anwendungsbeispiele im Rahmen der Aus- und Weiterbildung an der Pädagogischen Hochschule Zürich werden exemplarisch von Alain Desarzens und Susanne Ramsauer beschrieben. Nach der Erläuterung der wesentlichen Veränderungen im Schulfeld wird gezeigt, wie Studierende kognitiv und emotional für die systemischen Aspekte der Schule durch Soziodrama sensibilisiert werden. In einem weiteren Beispiel wird mittels psychodramatischer Rollenspiele die Erprobung des ,Ernstfalls Prüfung' dargestellt. Im zweiten Teil wird anhand eines, angeleiteten Sabbaticals` (im Text Intensivweiterbildung benannt) ein strukturell angelegter Perspektivenwechsel beschrieben, der insbesondere durch den Einsatz von soziometrischen Interventionen neue Erfahrungen und Resultate für Lehrerinnen und Lehrer ermöglicht.

Beatrix Wildt setzt sich in ihrem Beitrag mit Scham und Beschämung als Aspekte der Regulation sozialen Verhaltens auseinander. Dazu werden mehrere Formen von Scham unterschieden. An Beispielen aus Schule und Erziehungspraxis werden Probleme einer verbreiteten pädagogischen Praxis der Beschämung und deren Wirkungen aufgezeigt. Wie diese Praxis sich auswirken kann, wird in der psychodramatischen Arbeit mit LehrerInnen und SozialpädagogInnen deutlich und an Beispielen aus Beratung und Selbsterfahrung veranschaulicht. Es wird dargelegt, was das Psychodrama demgegenüber beabsichtigt und wie es zur Entwicklung einer professionellen pädagogischen Praxis beitragen kann, die an die Stelle von Beschämung Empowerment für ein gutes und gelingendes Leben setzt.

In einem außerschulischen Feld, dem Konfirmandenunterricht, kommen Kinder und Jugendliche aus sehr unterschiedlichen sozialen Hintergründen und Schulformen zusammen.,Integration“ ist im Konfirmandenunterricht nicht nur eines der inhaltlich zu vermittelnden religionspädagogischen Ziele, sondern eine durch Setting und Zusammensetzung der Lerngruppen gegebene Aufgabe. Die psychodramatischen Methoden und Arrangements eignen sich gut zur Erreichung dieser Lernziele. Dies wird exemplarisch an einer 
Unterrichtseinheit zu der biblischen Außenseiter-Geschichte ,Zachäus der Zöllner'von Achim Ludwig ausgearbeitet.

Mit Blick auf die Hochschule wird von Oliver Reis und Birgit Szczyrba die Frage bearbeitet, wie das Feld der wissenschaftlichen Promotionsbetreuung strukturiert ist. Was weiß man über die Beziehungen und Rollen zwischen Promovierenden als zukünftige ,Wissenschaftselite " und ihren BetreuerInnen? Warum scheitern so viele Promotionsvorhaben an der Betreuung? Der Beitrag befasst sich mit Rollenkonfigurationen in der Doktorandenbetreuung und untersucht, aus welchen Überzeugungen und Zielen sich diese speisen. Außerdem beschreibt der Beitrag eine auf den Erkenntnissen einer Studie basierende Weiterbildung, die auf die Erweiterung der Beraterqualifikation für wissenschaftliche BetreuerInnen zielt.

Die Hochschule bleibt im Fokus, wenn Johannes Wildt die Erteilung der Lehrbefähigung, die Hochschullehrenden mit der Berufung bescheinigt wird, auf den Prüfstand stellt: Die mit der Berufung verbriefte Lehrbefähigung ist an die Lizenz gebunden, den Bildungsprozess, das Lernen der Studierenden ,in Ordnung ${ }^{`} \mathrm{zu}$ bringen, d.h. zu unterrichten, auszurichten, anzuleiten zu beraten, anzuregen - welcher Lehrauffassung man auch immer anhängt. Kaum hat man je aus deutschen Hochschulen vernommen, dass Habilitationen oder Berufungen an der Feststellung mangelnder Eignung für die Lehre gescheitert seien. Umfangen vom Mythos des ,Ausgelernten“ stehen nun aber Hochschullehrende vor neuen und schwierigen Herausforderungen. Psychodrama kann in hochschuldidaktischer Weiterbildung und Beratung die Rollenkonserven von ,Ausgelernten“ in neue Handlungsmöglichkeiten radikal umwandeln.

Bei Ulrike Kroneck wird das leere Blatt zur Bühne: Wie das Psychodrama und sein methodisches Handwerkzeug genutzt werden kann, um Schreibprozesse zu initiieren und Texte zu kreieren, umreißt sie mit Übungen zum gezielten Perspektivenwechsel und Rollentausch. Das ,innere Personal' wird auf dem Papier lebendig. Die Szene wird zum charakteristischen Strukturmerkmal eines Textes und der schriftliche Dialog hilft, die im Text aufscheinenden Beziehungen der Menschen untereinander zu verdeutlichen. Die innere Wahrheit, nicht das Faktische, rückt in den Mittelpunkt.

Der Beitrag von Stefan Gunkel erörtert als „Der andere Artikel“ den essentiellen Kern und die Wirkung psychodramatischer Inszenierungen. Dabei stellt er einen starken Bezug zur einschlägigen wissenschaftlichen Literatur her. Gunkel zeigt, dass spielerisches Handeln in Rollen und die Nutzung verschiedener Techniken (Spiegel, Doppel usw.) explizite Rollenübernahme im Sinne des symbolischen Interaktionismus sind. Via Rollenspiel werden multiple Perspektiven in Bezug auf Sachverhalte oder Personen eingenommen, was nicht nur kognitive Dezentrierung und eine Erweiterung von Standpunkten mit sich bringt. Auch ein Zugewinn emotionaler Einfühlung im Sinne eines Empathietrainings wird damit möglich. Die Erkenntnis der Standortabhängigkeit von Sichtweisen und Gefühlen fördert die soziale Sensibilität und stärkt die interpersonelle Handlungskompetenz. So lassen sich mittels Psychodrama im Feld der Bildung und Erziehung v. a. interaktionelle Vorgänge lebendig erforschen. 